\title{
Validity of the Manchester Triage System in patients with sepsis presenting at the ED: a first assessment
}

\author{
Ingo Gräff, ${ }^{1}$ Bernd Goldschmidt, ${ }^{2}$ Procula Glien, ${ }^{1}$ Ramona C Dolscheid-Pommerich, ${ }^{3}$ \\ Rolf Fimmers, ${ }^{4}$ Daniel Grigutsch ${ }^{5}$
}

'Emergency Department, University Hospital Bonn, Bonn, Germany

${ }^{2}$ Department of Process Management, University Hospital Bonn, Bonn, Germany ${ }^{3}$ Department of Clinical Chemistry and Clinical Pharmacology, University Hospital Bonn, Bonn, Germany ${ }^{4}$ Institute for Medical Biometry, Informatics and Epidemiology, University Hospital Bonn, Bonn, Germany

${ }^{5}$ Department of Anesthesiology, University Hospital Bonn, Bonn, Germany

Correspondence to Dr Ingo Gräff, Emergency Department, University Hospital Bonn, Sigmund-Freud-Str. 25, Bonn 53127, Germany; info@medingo.de

Received 13 August 2015 Revised 23 November 2016 Accepted 27 November 2016 Published Online First 19 December 2016

\section{ABSTRACT}

Background The Manchester Triage System (MTS) does not have a specific presentational flow chart for sepsis. The goal of this investigation was to determine adequacy of acuity assignment for patients with sepsis presenting at the ED and triaged using the MTS.

Materials and methods This retrospective analysis included patients $>16$ presenting to an ED in Bonn, Germany, on the first 12 days of each month between June 2012 and March 2014. Patients were classified into one of three septic groups, or no sepsis. For those with sepsis, adequacy of acuity assignment was based on the criteria of the first consensus conference of the American College of Chest Physicians and Society of Critical Care Medicine, first published in 1992. Adequacy of prioritisation is expressed as sensitivity and likelihood ratio (LR-).

Results Among 20836 patients evaluated, 801 (3.8\%) were septic; of these, 581 (72.5\%) had sepsis, 194 (24.2\%) had severe sepsis and 26 (3.2\%) had severe sepsis with circulation dysfunction. Patients who met the criteria for sepsis were correctly prioritised with a sensitivity of $70.4 \%(95 \% \mathrm{Cl} 66.5$ to 74.0$)$. The LR- was 0.628 (95\% Cl 0.564 to 0.698). Patients with severe sepsis were appropriately prioritised with a sensitivity of $84.5 \%(95 \% \mathrm{Cl} 78.1$ to 89.4$)$, and LR- was 0.330 ( $95 \% \mathrm{Cl} 0.243$ to 0.450$)$. In the group with severe sepsis and circulation dysfunction, sensitivity of MTS was $61.5 \%$ (95\% Cl 39.3 to 79.8$)$, and LR- was 0.466 (95\% Cl 0.286 to 0.757$)$.

Conclusions The MTS has some weaknesses regarding priority levels in emergency patients with septic illness. Overall, target key symptoms (discriminators) which aim at identifying systemic infection and ascertaining vital parameters are insufficiently considered.

\section{INTRODUCTION}

Current epidemiological data for the German population show an incidence of 106/100 000 for sepsis, $84 / 100000$ for severe sepsis and 23/ 100000 for septic shock. ${ }^{1}$ Internationally, it is assumed that approximately two-thirds of all patients with sepsis are admitted through the ED. ${ }^{2}$ Data from the USA show a $0.7 \%$ prevalence for patients with severe sepsis for all ED cases, that is, 571000 patients annually. ${ }^{3}$

Early identification of patients with sepsis, ideally on arrival at the ED, is essential for effective therapy and for improving clinical outcomes. Chaudhary et $a l^{4}$ consider sepsis to be the same level of urgency as other time-sensitive diseases, such as ST elevation myocardial infarction and stroke. With every hour of delay in antibiotic

\section{Key messages}

What is already known on this subject? There is no presentational flow chart in the Manchester Triage System (MTS) for patients with systemic infection or sepsis. In contrast to this, there are specially developed scoring systems available such as the Mortality in Emergency Department Sepsis Score or the National Early Warning Score, which is broadly used in the UK. Using these, the specific retrieval of sepsis symptoms or vital parameters, critically ill patients can be assessed at triage with higher reliability.

\section{What this study adds?}

In this retrospective study of 20836 randomly selected patients, 801 had sepsis on arrival at the ED. Using the MTS, $30 \%$ of patients with sepsis were erroneously categorised to low acuity priorities. Modifications to the MTS should be pursued to improve categorisation of priority levels.

therapy in cases of sepsis, survival decreases by $7.6 \%$. In a recent prospective study, Machado et $a l^{5}$ found that a delay in diagnosis and, consequentially, therapy onset, increases early mortality. Substantially lower mortality was found among ED patients who had received antibiotic treatment within the first hour. ${ }^{67}$ Similar to polytrauma management, a 'golden hour' of therapy has therefore been postulated for sepsis. ${ }^{4}$

It has become apparent that EDs play a key role in the early identification of patients with sepsis. For these cases, the operation of triage systems and their embedded filter functions during the triage decision-making process is crucial. On account of this, Wrede et $a l^{8}$ call for studies of currently implemented initial assessment systems such as the Manchester Triage System (MTS). The MTS (second edition) has found widespread use in EDs across Europe and the broadest application in German EDs.

The German MTS uses presentational flow charts of complaint complexes backed by 50 algorithms (such as 'abdominal pain in an adult' or for unspecific symptoms, such as 'unwell adult') to target key symptoms (so-called 'discriminators') and to allocate the patient to one of five levels of priority. These priority levels indicate the maximal time allowed from the patient's arrival until a doctor should see the patient. Patients triaged in 
the highest category (red) are in need of immediate treatment. The next two categories (orange and yellow) have longer recommended time allowances (10 and $30 \mathrm{~min}$, respectively). The two lowest categories (green and blue) have the longest recommended time allowances of 90 and 120 min. $^{9}$ In the original English MTS version, the time allowances are longer for triage levels 'yellow', 'green' (1.5 times) and 'blue' (twice as long) as in the German version. In addition, there are 53 presentational flow charts in the English version (three additional paediatric algorithms). Since 2008, the MTS has only undergone a few revisions, which speaks for a stable triage system.

A special presentational flow chart specifically for sepsis does not exist within the MTS, and the MTS has not been specifically validated for its ability to adequately prioritise patients with sepsis. We sought to answer the question of how sensitive the MTS is at prioritising patients who are septic, severely septic or have severe sepsis with circulation dysfunction. The identification of potential weaknesses in the MTS for these patients is warranted, and possible steps for improvement are enumerated here. For the purposes of this study, we used the S-2K guidelines of the German sepsis society, valid since $2005 .{ }^{10}$ These are based on the SIRS criteria, which were defined for the first time by the American College of Chest Physicians (ACCP) and Society of Critical Care Medicine (SCCM) in 1992. ${ }^{11}$ While there is a newer definition of sepsis, it has not yet been operationalised and most EDs continue to use the SIRS criteria to screen patients for sepsis. ${ }^{12}$

\section{MATERIALS AND METHODS Setting}

This was a single-centre retrospective observational study performed at the ED of the University Hospital Bonn, Germany. The jurisdiction of the ED stretches beyond the city limits of Bonn to include surrounding districts, with a total of approximately 1 million residents. The ED of the University Hospital Bonn has the highest level of visits compared with other EDs in the neighbouring areas (including cardiac arrest centers and trauma centers) and annually treats approximately 30000 emergency patients. Gynaecologic, obstetric and paediatric emergencies up to age 14 (with the exception of traumatised children and children with ENT problems) are cared for in nearby clinics. Triage in the ED at the University Hospital Bonn is a standardised process, which is clearly outlined in quality guidelines. Each patient presenting as an emergency case is first seen by a specially trained nurse and triaged according to the MTS (triage protocol). All 24 triage nurses were trained in a two and one half day in-house schooling for the MTS prior to working in the ED. Since 2009, the quality of triage has regularly been evaluated via audit three times a year. Since 2012, the team has had an MTS trainer in its ranks who is responsible for the supervision of the triage by MTS. For the rare case in which the triage nurse disagrees with the MTS assignment, the nurse can discuss this directly with the medical doctor on duty. Furthermore, contact with the German MTS reference group is always possible in real time.

The observation period spanned from 1 July 2012 to 14 March 2014. This period was chosen because in May 2012, the digital form for recording triage data was changed from selfprogrammed solution to a commercial hospital information system. During the evaluation period, there was no change in observational conditions (eg, number of nurses or workflow), MTS training and supervision was also unchanged. During the observation, there were 53839 visits, which would not have been feasible to review. For this reason, a pseudo-randomisation procedure was chosen: patients presenting on the first 12 days of each month during the observation period were reviewed. In addition, because The S-2K guidelines of the German sepsis society do not apply to patients under 16 years of age, patients under 16 were excluded from analysis.

\section{Identifying patients according to the sepsis guidelines}

The sepsis category was retrospectively determined using a computer algorithm. Only examinations and investigations taken at the time of admission to the ED were considered, even if some of the results were available later. Using this sepsis screening tool, the patient triage notes were analysed for positive SIRS criteria. These were temperature, HR and RR. The leucocyte value, as the fourth SIRS criterion, was determined from the blood sampling taken in the ED. For the presence of sepsis, at least two positive SIRS criteria and an additional infection must be present. The severe sepsis is also associated with acute organ failure. ${ }^{10}$ Detection of infection was by positive blood culture (blood sample taken at the time of admission) or by clinical signs of infection. Clinical signs of infection at the time of admission were retrospectively evaluated from the patient's data. For this purpose, the screening tool was used to search for infections by predefined keywords such as abscess, swelling, pus, erysipelas, infection, confusion, and so on. The acute organ failure was also evaluated by keywords and lab results using the computer algorithm. Organ failure was diagnosed in the presence of acute encephalopathy, oxygenation problems, disorders of the thrombocytes, impaired renal function or an acid-base imbalance. By definition, it is only septic shock if hypotension occurs longer than an hour or vasopressors are necessary after fluid resuscitation. Patients in our group with an RR $\leq 90 \mathrm{~mm} \mathrm{Hg}$, who did not respond to a fluid bolus and required vasopressors, have been classified as severe sepsis with circulation dysfunction.

All cases classified by the sepsis screening tool into one of the three sepsis categories were checked by an emergency physician who reviewed, and triage data were analysed for patient history, narrative comments, symptoms, vital signs and laboratory data. Identification by the algorithm was considered correct if an infection was present and associated with SIRS criteria. A false positive assignment by the computer programme was excluded. For example, mental confusion caused by trauma was not been counted as a sign of infection.

The 'healthy' collective (ie, 'no sepsis') contains patients without sepsis and all patients with an infection who did not fulfil the formal criteria of the sepsis definition.

\section{Determination of cut-off values}

Based on the issues mentioned above and the crucial 'golden hour of sepsis', it is assumed that the determination of the triage level has an immediate effect on mortality. Allocation of patients who are septic and severely septic to MTS triage categories of 'yellow', 'orange' or 'red' was considered correct in this study. MTS category 'green' or 'blue' was judged to be inadequate prioritisations. Patients with severe sepsis with circulation dysfunction were considered adequately categorised only when allocated to 'orange' or 'red'. Sensitivity was the proportion of patients with sepsis correctly allocated. The negative predictive value (NPV) indicates the proportion of patients with sepsis undertriaged.

\section{MTS and sepsis guidelines-how do the two go together?}

There is no provision for a structured assessment of the vital signs within the MTS for determining triage level. In addition, 
when vital signs are taken, there are discrepancies between the SIRS criteria and the discriminator criteria on which MTS is based (table 1). Whereas SIRS criteria uses a cut-off of RR $\geq 20$ breaths per minute, MTS describes inadequate breathing or acutely short of breath'. SIRS criterion is a HR of $\geq 90 \mathrm{bpm}$, whereas for the 'orange' MTS category, the cut-off value is $>120 \mathrm{bpm}$. SIRS temperature criteria are $\geq 38^{\circ} \mathrm{C}$ or $\leq 36^{\circ} \mathrm{C}$. To be triaged as 'orange' within MTS, the patient has to have a body temperature of $>41^{\circ} \mathrm{C}$ or $<35^{\circ} \mathrm{C}$. For 'yellow', MTS stipulates a temperature of $>38.5^{\circ} \mathrm{C}$. Therefore, there are two temperature ranges in the MTS which would not contribute to the SIRS criteria $\left(\geq 35^{\circ} \mathrm{C}\right.$ or $\leq 36^{\circ} \mathrm{C}$ and $\geq 38 \mathrm{C}^{\circ}$ or $\left.\leq 38.5^{\circ} \mathrm{C}\right)$. BP is mentioned as a discriminator in only one of the 50 presentational flow charts (pregnancy complications). While it is not an SIRS criteria, it is a marker of severe sepsis with circulatory dysfunction.

\section{Outcome}

The primary outcome was the proportion of patients with any sepsis who were categorised in one of the three highest priorities (red, orange and yellow) by the MTS and those who were missed. A secondary outcome was the proportion of patients with severe sepsis or severe sepsis with circulatory function who were not categorised as either red or orange.

\section{Statistical analysis}

Statistical evaluation was conducted using SAS (V.9.2; SAS Institute, Cary, North Carolina, USA). Results are presented as means, SD with 95\% CIs and numbers or percentages, respectively. A $\chi^{2}$ test was used for comparison of gender at baseline. For the quantitative parameters, a one-way analysis of variance was conducted. Correlation between triage category and number of patients with sepsis, severe sepsis and severe sepsis with circulation dysfunction was assessed with the Cochran-Armitage Trend test. Test characteristics of sensitivity, NPV and likelihood ratio (LR-) were calculated.

\section{Ethics statement}

The German General Medical Council explicitly excludes retrospective studies from approval by the ethics committee in their code of medical ethics (article 15/1) (http://www.aekno.de/page. asp?pageID $=57 \#$ 15). Furthermore, as stipulated in article six of the German Data Protection Act (https://recht.nrw.de/lmi/ owa/br_text_anzeigen?v_id=10000000000000000495\#), the physician may use existing patient data for retrospective analyses without explicitly asking for the consent of patients. All collected clinical data evaluated in this study were fully anonymised before analysis. Therefore, according to prior agreement with

Table 1 Discrepancies between SIRS criteria and MTS discriminators

\begin{tabular}{|c|c|c|}
\hline Vital sign & MTS orange & ACCP/SCCM \\
\hline $\begin{array}{l}\text { RR (breaths per } \\
\text { minute) }\end{array}$ & $\begin{array}{l}\text { 'Inadequate breathing or } \\
\text { acutely short of breath' }\end{array}$ & $\geq 20$ \\
\hline HR (bpm) & $>120$ & $\geq 90$ \\
\hline Temperature $\left({ }^{\circ} \mathrm{C}\right)$ & $\begin{array}{l}<35 \text { or }>41 \\
>38.5 \text { (yellow) }\end{array}$ & $\leq 36$ or $\geq 38$ \\
\hline $\mathrm{BP}(\mathrm{mm} \mathrm{Hg})$ & Only mentioned for pregnancy & $\begin{array}{l}\leq 90 \text { systolic for } \\
\text { 'circulatory dysfunction' }\end{array}$ \\
\hline
\end{tabular}

the local ethics committee and the data protection officer appointed by the University Clinics Bonn, verbal or written informed consent was not obtained. The study design is consistent with the Declaration of Helsinki.

\section{RESULTS}

The pseudo-randomisation procedure yielded 20836 patient visits for study. Of these, 801 (3.8\%) were septic: $581(72.5 \%)$ had sepsis, 194 (24.2\%) had severe sepsis and 26 (3.2\%) had severe sepsis with circulation dysfunction. Demographic characteristics, vital signs and laboratory parameters of the entire cohort are shown in table 2 .

\section{Patients with sepsis}

Among all 801 patients with sepsis, 564 patients (70.4\%) were allocated to 'yellow', 'orange' or 'red', while 237 were mistriaged to either green (229 patients, $28.6 \%$ of the septic group) or blue (8 patients, $1 \%$ of the septic group) for an NPV of $97.6 \% \quad(95 \%$ CI 97.2 to 97.9) (table 3). A correlation $(p<0.001)$ was found between triage level and increasing number of patients with sepsis. Sensitivity for assignment of patients to an appropriate MTS triage level in the three highest categories was $70.4 \%$ (sensitivity) (95\% CI 66.5 to 74.0 ), the NPV was $97.6 \%$ (95\% CI 97.2 to 97.9) and LR- =0.628 (95\% CI 0.564 to 0.698$)$. Among patients with sepsis who were correctly classified, the most commonly used presentational flow chart was 'shortness of breath in adults'.

For patients with sepsis classified 'green,' the most commonly used flow charts were 'unwell adult', 'sore throat' and 'ear problems'. Regarding SIRS parameters, the HRs of 179 patients fell within the range between 90 and $120 \mathrm{bpm}$, while 107 patients had body temperatures in the range $\geq 35^{\circ} \mathrm{C}$ to $\leq 36^{\circ} \mathrm{C}$ and 27 patients in the range $\geq 38^{\circ} \mathrm{C}$ to $\leq 38.5^{\circ} \mathrm{C}$. Twelve patients had an $\mathrm{RR}$ of $\geq 20$ breaths per minute. Laboratory tests revealed leukocytosis in 72 patients, leucopenia in eight patients and positive blood culture in eight patients.

For patients with sepsis assigned to category 'blue', the most frequently applied presentational flow charts were 'unwell adult' and 'general complaints'. Five misclassified patients with sepsis had HRs between 90 and $120 \mathrm{bpm}$. Three misclassified patients had body temperature range between $35^{\circ} \mathrm{C}$ and $36^{\circ} \mathrm{C}$. One patient had an RR of $\geq 20$ breaths per minute (table 4). Laboratory tests revealed leukocytosis in three patients.

\section{Patients with severe sepsis}

Among the 220 patients with severe sepsis, 186 (84.55\%) were correctly classified as 'yellow', 'orange' or 'red'. Of the others, 33 patients $(15 \%)$ were prioritised to the category 'green' and 1 patient $(0.45 \%)$ was assigned to the lowest category of 'blue'. The sensitivity of MTS for severe sepsis was $84.5 \%$ (95\% CI 78.1 to 89.4 ), the NPV $99.6 \%$ (95\% CI 99.5 to 99.8$)$ and the LR - was 0.330 (95\% CI 0.243 to 0.450$)$.

For patients with severe sepsis who were correctly classified, the most commonly used presentational flow chart was 'shortness of breath in adults'. For the triage categories 'green' and 'blue', the most frequently applied presentational flow chart was 'unwell adult'. Among the severe sepsis patients allocated to the 'green' category, 23 fell within the crucial HR range of 90-120 bpm, while 9 patients had a body temperature in the range $\geq 35^{\circ}$ $\mathrm{C}$ to $\leq 36^{\circ} \mathrm{C}$ and 10 in the range $\geq 38^{\circ} \mathrm{C}$ to $\leq 38.5^{\circ} \mathrm{C}$. Four patients had an RR of $\geq 20$ breaths per minute. Laboratory tests revealed leukocytosis in 13 patients, leucopenia in 9 patients and positive blood culture in 7 patients. Furthermore, patients of the 'green' category showed signs of organ failure on arrival 


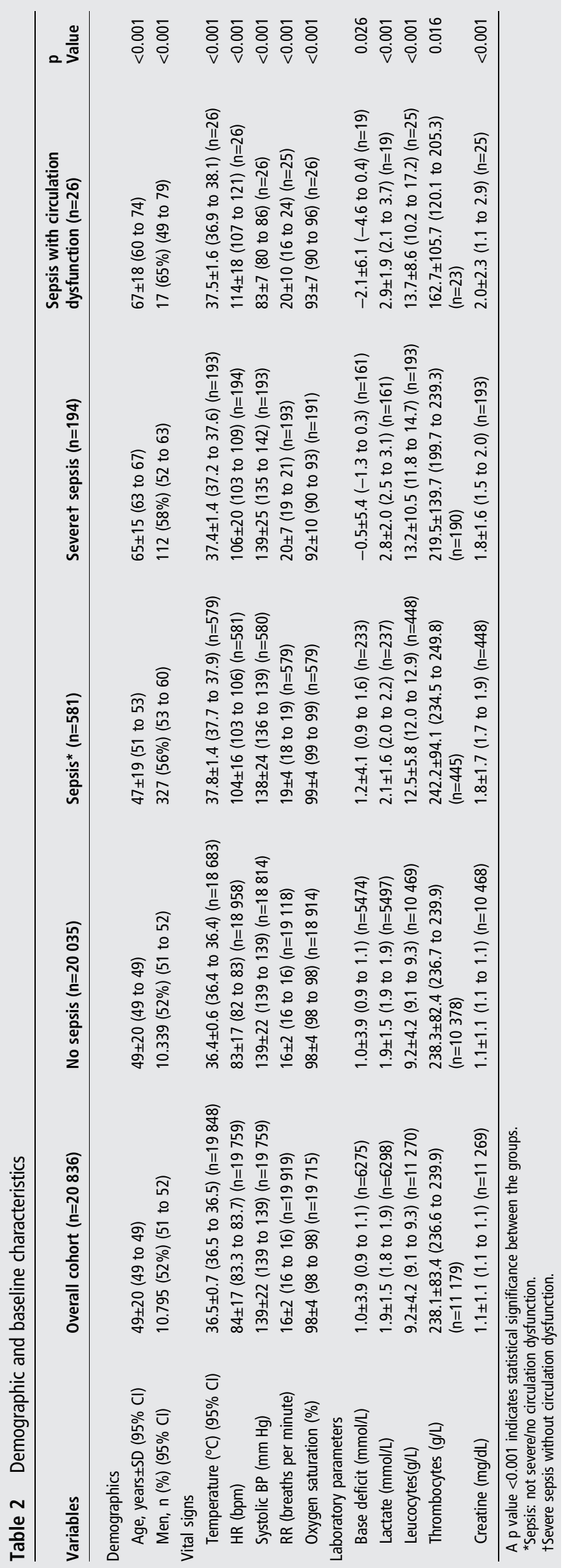

at the ED. These were oxygenations problem and mental confusion. For the one case severe sepsis allocated to the 'blue' category, the HR fell into the range 90-120 bpm (table 4).

\section{Patients with severe sepsis and circulation dysfunction}

Among 26 patients with severe sepsis and circulation dysfunction, 16 patients $(61.5 \%)$ were adequately prioritised to the categories 'orange' and 'red' (table 3 ). The most commonly applied presentational flow chart for this group was 'unwell adult'. There was a significant correlation between triage level and number of patients with severe sepsis and circulation dysfunction $(\mathrm{p}<0.001)$. The sensitivity of MTS was $61.5 \%(95 \%$ CI 39.3 to 79.8 ), the NPV $99.9 \%$ (95\% CI 99.9 to 100.0 ) and LR - 0.466 (95\% CI 0.286 to 0.757 ).

Nine patients with sepsis with circulation dysfunction (34.6\%) were assigned to category 'yellow', one patient (3.8\%) to category 'green' and no patient was triaged into category 'blue'. Seven patients assigned to 'yellow' had HRs within the range of 90-120 bpm, three patients had a body temperature between $38^{\circ} \mathrm{C}$ and $38.5^{\circ} \mathrm{C}$ and one patient had a body temperature between $35^{\circ} \mathrm{C}$ and $36^{\circ} \mathrm{C}$. Three patients had an RR of $\geq 20$ breaths per minute and nine patients had a $\mathrm{BP}<90 \mathrm{~mm} \mathrm{Hg}$. Regarding other SIRS parameters, leukocytosis was revealed via blood chemistry tests in four patients, and leucopenia in three patients. Four of the 'yellow' patients showed signs of organ failure (oxygenation problems) on arrival at the ED.

The one 'green' patient fell within the crucial HR range of 90 $120 \mathrm{bpm}$, his body temperature was between $35^{\circ} \mathrm{C}$ and $36^{\circ} \mathrm{C}$, RR was 20 beats per minute and BP was $\leq 90 \mathrm{~mm} \mathrm{Hg}$ (table 4).

\section{DISCUSSION}

Early recognition and application of basic therapies is crucial for the appropriate and efficient management of sepsis. The aim of this study was to assess the validity of MTS as a priority assessment tool, specifically in patients with sepsis, severe sepsis and severe sepsis with circulation dysfunction. We found that there is potential for improvement in the allocation of priority among adult sepsis patients presenting at the ED. This is consistent with a prior study showing that MTS leads to undertriage in critically ill children. ${ }^{13}$

A study by Chamberlain $e t ~ a l^{14}$ examined patients with severe sepsis with the Australasian Triage Scale (ATS). Like the MTS, the ATS is also a five-stage triage system and, similar to the MTS, it uses individual clinical descriptors. Also in this study the classification of sepsis was made retrospectively, based on the sepsis guidelines. The overall sensitivity of the ATS to identify severe sepsis was $71 \%$. The authors conclude that the ATS lacks clinical efficacy and safety without further education or quality improvement strategies targeted to the identification of severe sepsis.

Geier $e t a l^{15}$ evaluated the Emergency Severity Index (ESI) in a prospective study in patients with severe sepsis and septic shock. The working group found a sensitivity of $70.8 \%$, the NPV was $62.3 \%$ and the LR- was $64 \%$. Like the MTS, the ESI is a five-stage triage system. This may also be related to the lack of a uniform requirement for vital signs. If the patient is not classified in ESI 1 or 2 and requires less than two resources, there is no requirement for vital parameter measurement, although many departments using ESI perform vital signs on all patients. In addition, the cut-off value of the HR ( $>100 \mathrm{bpm})$ is higher than that of the SIRS criteria (a decision made by the developers to avoid too many false positives) and the BP is completely missing in the ESI 'vitals danger zone'. ${ }^{16}$ 
Table 3 Frequency distribution of Manchester Triage System (MTS) levels within diagnostic groups

\begin{tabular}{|c|c|c|c|c|c|c|}
\hline & $\begin{array}{l}\text { Overall cohort } \\
(n=20836)(\%)\end{array}$ & $\begin{array}{l}\text { No sepsis } \\
(n=20035)(\%)\end{array}$ & $\begin{array}{l}\text { All sepsis } \\
(n=801)(\%)\end{array}$ & $\begin{array}{l}\text { Sepsis* } \\
(n=581)(\%)\end{array}$ & $\begin{array}{l}\text { Severe sepsist } \\
(n=194)(\%)\end{array}$ & $\begin{array}{l}\text { Sepsis with circulation } \\
\text { dysfunction }(n=26)(\%)\end{array}$ \\
\hline Red & 338 (1.6) & 316 (1.6) & $22(2.7)$ & $12(2.1)$ & 7 (3.6) & $3(11.5)$ \\
\hline Orange & 3298 (15.8) & 3118 (15.6) & $180(22.5)$ & $89(15.3)$ & $78(40.2)$ & $13(50)$ \\
\hline Yellow & $7524(36.1)$ & 7162 (35.7) & $362(45.2)$ & 277 (47.7) & $76(39.2)$ & 9 (34.6) \\
\hline Green & 8733 (41.9) & 8504 (42.4) & $229(28.6)$ & $196(33.7)$ & $32(16.5)$ & $61(3.8)$ \\
\hline Blue & $943(4.53)$ & $935(4.7)$ & $8(1.0)$ & $7(1.2)$ & $1(0.5)$ & $-(0.0)$ \\
\hline
\end{tabular}

Table 4 Presenting findings among patients mistriaged

\begin{tabular}{|c|c|c|c|c|c|c|}
\hline \multirow[b]{2}{*}{ MTS level } & \multicolumn{2}{|c|}{ Sepsis $(n=581)^{*}$} & \multicolumn{2}{|c|}{ Severe sepsis $(n=194) \dagger$} & \multicolumn{2}{|c|}{$\begin{array}{l}\text { Sepsis with circulation } \\
\text { dysfunction }(n=26)\end{array}$} \\
\hline & Green (\%) & Blue (\%) & Green (\%) & Blue (\%) & Yellow (\%) & Green (\%) \\
\hline \multicolumn{7}{|l|}{ Directly accessible at triage } \\
\hline \multicolumn{7}{|l|}{ SIRS criteria } \\
\hline$\geq 90 \mathrm{HR} \leq 120 \mathrm{bpm}$ & $179(30.80)$ & $5(0.86)$ & $23(11.85)$ & $1(0.51)$ & $7(26.92)$ & $1(3.84)$ \\
\hline$\geq 35^{\circ} \mathrm{C}$ Temp. $\leq 36^{\circ} \mathrm{C}$ & $107(18.41)$ & $3(0.51)$ & $9(4.63)$ & - & $1(3.84)$ & $1(3.84)$ \\
\hline$\geq 38^{\circ} \mathrm{C}$ Temp. $\leq 38.5^{\circ} \mathrm{C}$ & $27(4.64)$ & - & $10(5.15)$ & - & $3(11.53)$ & - \\
\hline $\mathrm{RR} \geq 20$ breaths per minute & $12(2.06)$ & $1(0.17)$ & $4(2.06)$ & - & $3(11.53)$ & $1(3.84)$ \\
\hline \multicolumn{7}{|l|}{ Additional criteria for sepsis } \\
\hline Oxygenation problems & - & - & $11(5.67)$ & - & $2(7.69)$ & - \\
\hline Mental confusion & - & - & $2(1.03)$ & - & - & - \\
\hline Systolic BP $\leq 90$ mm Hg & - & - & - & - & $9(34.61)$ & $1(3.84)$ \\
\hline \multicolumn{7}{|l|}{ Retrospectively accessible } \\
\hline Leucocyte count $\geq 12000 / \mathrm{mm}^{3}$ & 72 (12.39) & $3(0.51)$ & $13(6.70)$ & $1(0.51)$ & $4(15.38)$ & $1(3.84)$ \\
\hline Leucocyte count $\leq 4000 / \mathrm{mm}^{3}$ & $8(1.37)$ & $1(0.17)$ & $9(4.63)$ & - & $3(11.53)$ & - \\
\hline Acid-base imbalance & - & - & $16(8.24)$ & $1(0.51)$ & $2(7.69)$ & - \\
\hline Positive blood culture & $8(1.37)$ & - & $7(3.60)$ & $1(0.51)$ & $4(15.38)$ & - \\
\hline
\end{tabular}

*Sepsis: not severe/no circulation dysfunction.

tSevere sepsis without circulation dysfunction.

MTS, Manchester Triage System.

National Early Warning Score (NEWS) is the scoring system used for standardising the assessment of acute illness severity among undifferentiated patients, in the UK. Keep et $a l^{17}$ showed that a NEWS of 3 or more at ED triage may be the trigger to systematically screen the patient for severe sepsis, which may ultimately lead to early recognition and treatment. A NEWS of 3 or more at ED triage has a sensitivity of $92.6 \%$ (95\% CI $74.2 \%$ to $98.7 \%$ ) and a specificity of $77 \%$ (95\% CI $72.8 \%$ to $80.6 \%)$ to detect patients at risk for severe sepsis at ED triage. The NEWS is based on its own independent systems and is different in its constellation from MTS. Apparently, the standardised evaluation of the vital signs as part of determining the treatment priority increases the sensitivity for sepsis patients.

The low sensitivity of $61 \%$ for the sepsis group with circulatory dysfunction ascertained in this study indicates a weakness in MTS for the clinical routine. The explanation of the discriminator shock in the MTS category 'red' is 'inadequate delivery of oxygen to the tissues'. It is based on clinical symptoms such as sweating, pallor, tachycardia and hypotonia and certainly identifies patients with haemorrhagic shock and the necessity of life-saving intervention. Within the context of a systemic infection, the lack of a blood pressure cut-off value may result in inadequate triage of these patients. The inclusion of blood pressure with a cut-off value for the MTS category 'orange' would allow an increase in sensitivity in our collective of patients with sepsis with circulation dysfunction. The routinely assessed MTS vital signs need to be recalibrated to the cut-off values of the current SIRS criteria (both of these issues are taken into account in NEWS).

Furthermore, the MTS needs to assess symptoms that indicate systemic infections. In the authors' opinion, greater consideration of relevant organ dysfunction/SIRS criteria at the first point of contact during triage is necessary. Figure 1 shows two cases which underline this difficulty. Chamberlain et $a l^{14}$ also call for the inclusion of more infection-related discriminators in a five-level triage system. Because of the fact that many elderly patients with systemic infection do not have a fever on arrival, such unspecific symptoms such as chills, disorientation, apathy, lack of appetite, mottled skin and diarrhoea also ought to be taken into consideration in MTS. ${ }^{18}$

The results of this study compel the further development of the MTS. The next step should be the development of additional discriminators or a special presentational flow chart, in agreement with the international MTS Reference Group. Parallel to this, rectification of the discrepancies between HR 
Let us look at the example of a 67-year-old woman arriving at the emergency department with flu-like symptoms that have been persisting for 2 days, cough, yellowish secretion and a body temperature of $38.4^{\circ} \mathrm{C}$. Her respiration rate is 20 breaths per minute, saturation is $97 \%$, her heart rate is $91 \mathrm{bpm}$. GCS is 15 and VAS is 3 . Based on the sepsis guidelines, it must be assumed admission at the emergency department that this patient is suffering from sepsis.

Of concern is also the following constellation: When a patient presents with infection after, eg, arthroscopic surgery of the knee joint, the nurse will most likely apply the presentational flow chart 'limb problem'. In this presentational flow chart, SIRS criterions are insufficiently considered by MTS.

Figure 1 Two case scenarios. These two examples highlight the discrepancy between the sepsis guidelines and their SIRS criteria and the Manchester Triage System (MTS) allocation to triage categories. GCS, Glasgow Coma Scale; VAS, Visual Analogue Scale.

and body temperature cut-offs in MTS and the ACCP/SCCM SIRS criteria, extension of BP cut-offs to presentational flow charts other than pregnancy and the inclusion of RR cut-offs are essential. All modifications should be directed by a scientific board and evaluated via multicentre prospective studies.

Although this study was conducted using the German version of the MTS, the core messages are entirely transferable to the English version of MTS, especially because for the MTS levels 'yellow', 'green' and 'blue', the maximum permissible time to first physician contact is even longer than in the German version. Had we used the Sepsis Guidelines of the Surviving Sepsis Campaign, the results would not be substantially different as these are almost identical to the ACCP/SCCM criteria). ${ }^{19}$ It ought to be mentioned that there is a new definition of sepsis (sepsis 3) since the beginning of $2016 .{ }^{12}$ Even though SIRS as a criteria for the diagnosis of sepsis has been dropped, parameters of organ dysfunction, such as mental changes, breathing frequency and BP, are still used in the case of suspected sepsis. In addition, clinical infection parameters such as temperature, leucocyte count, biomarkers or HR still play a role in diagnosis.

\section{Limitations}

The identification of patients with sepsis, severe sepsis and severe sepsis with circulation dysfunction was performed with a screening tool; the subsequent verification of an underlying

Table 5 Weaknesses of the Manchester Triage System (MTS) regarding priority levels in emergency patients with septic illness

(a) Sensitivity and specificity of MTS in patients with sepsis, severe sepsis and severe sepsis with circulation dysfunction are moderate to adequate.

(b) There are no cut-off values for the two discriminators RR and BP in MTS

(c) Discriminators for body temperature and HR are not congruent with the SIRS parameter values of the sepsis guidelines.

(d) MTS presentational flow charts like 'limb problem' consider SIRS criterions insufficiently.

(e) Body temperature is included in less than half of the MTS 50 presentational flow charts.

(f) HR is included in only eight presentational flow charts. systemic infection was carried out by an emergency physician. Both methods are based on retrospective analysis of patient files without the actual clinical presentation of the emergency patient. Although the assignment to the three levels of sepsis was based exclusively on the clearly defined SIRS criteria and parameters from the patient files were unambiguous, a certain percentage of systemic infections may have been misidentified. Also, a small percentage of emergency patients may have had sepsis without presenting sufficient positive SIRS criteria. As these patients could not be evaluated separately in this study, they may have been wrongly included into the group of patients without sepsis criteria. This would only have affected specificity, which is not really the concern of this study as there are many other reasons that patients could be made high acuity unrelated to sepsis. The comparability with previous studies is not diminished by this fact since they were conducted under the same conditions. A further limitation is that the method used to measure body temperature was not in accordance with the specification of the criteria catalogue of the ACCP/SCCM consensus conference. The standardised temperature measurement method at the ED is the tympanic infrared thermometer measurement, which may have introduced significant potential for inaccuracy. However, Jefferies et $a l^{20}$ showed in a systematic review that, compared with rectal or oral measurement methods, tympanic infrared measurement is to be recommended in critically ill patients. Ultimately, the group of correctly prioritised emergency patients was not separately evaluated.

\section{CONCLUSIONS}

In its present version, the MTS shows some significant potential of improvement regarding priority levels in emergency patients with septic illness (table 5). The lack of consideration of vital signs cut-off values and the SIRS criteria of the criteria catalogue of the ACCP/SCCM consensus conference could lead to inadequate prioritisation. Modifications and multicentre prospective studies should be pursued to improve categorisation of priority levels.

Contributors IG, BG, PG, RCD-P and DG: conceived the study, designed the trial and drafted the article. IG, BG, RF and DG: analysed the data. IG, BG, PG, RCD-P, RF and DG: Approved the final version.

\section{Competing interests None declared.}

Ethics approval Following consultation with the chairman of the local ethics committee (K. Racké, MD, PhD, Professor, University Clinics Bonn) permission was obtained to analyse the data evaluated in this study without approval by the ethics committee since the analysis was purely retrospective. The German General Medical Council explicitly excludes retrospective studies from approval by the ethics committee in their code of medical ethics (article 15/1) (http://www.aekno.de/page. asp?pagelD=57\#_15). Furthermore, as stipulated in article six of the German Data Protection Act (https://recht.nrw.de/lmi/owa/br_text_anzeigen?v_ $\mathrm{id}=10000000000000000495 \#$ ), the physician may use existing patient data for retrospective analyses without explicitly asking for the consent of patients. All collected clinical data evaluated in this study were fully anonymised before analysis. Therefore, according to prior agreement with the local ethics committee and the data protection officer appointed by the University Clinics Bonn, verbal or written informed consent was not obtained. The study design is consistent with the Declaration of Helsinki.

Provenance and peer review Not commissioned; externally peer reviewed.

\section{REFERENCES}

1 Brunkhorst FM, Schmitz RP. Sepsis-Epidemiologie, Biomarker und mikrobiologische Diagnostik. Klinikarzt 2014;43:284-8.

2 World Sepsis Day_HOME_-[Internet]. (cited 29 March 2015). http://www. world-sepsis-day.org/?MET=HOME\&VLANGUAGE=DE

3 Wang HE, Shapiro NI, Angus DC, et al. National estimates of severe sepsis in United States emergency departments. Crit Care Med 2007;35:1928-36.

4 Chaudhary T, Hohenstein DC, Bayer O. Die goldene Stunde der Sepsis. Med Klin Intensivmed Notfmed 2014;109:104-8. 
5 Machado FR, Salomão R, Rigato 0, et al. Late recognition and illness severity are determinants of early death in severe septic patients. Clinics 2013;68:586-91.

6 Gaieski DF, Mikkelsen ME, Band RA, et al. Impact of time to antibiotics on survival in patients with severe sepsis or septic shock in whom early goal-directed therapy was initiated in the emergency department. Crit Care Med 2010;38:1045-53.

7 Jalili $\mathrm{M}$, Barzegari $\mathrm{H}$, Pourtabatabaei $\mathrm{N}$, et al. Effect of door-to-antibiotic time on mortality of patients with sepsis in emergency department: a prospective cohort study. Acta Med Iran 2013;51:454-60.

8 Wrede PDCE, Reinhart K. Sepsis in der Notfallmedizin. Notf Rettungsmedizin 2014; 17:707-17.

9 Mackway-Jones K. Ersteinschätzung in der Notaufnahme: das Manchester-Triage-System. Bern: Huber, 2011.

10 Reinhart K, Brunkhorst FM, Bone H-G, et al. Prevention, diagnosis, therapy and follow-up care of sepsis: 1st revision of S-2k guidelines of the German Sepsis Society (Deutsche Sepsis-Gesellschaft e.V. (DSG)) and the German Interdisciplinary Association of Intensive Care and Emergency Medicine (Deutsche Interdisziplinäre Vereinigung für Intensiv- und Notfallmedizin (DIVI)). Ger Med Sci 2010;8 (cited 29 March 2015). http://www.ncbi.nlm.nih.gov/pmc/articles/PMC2899863/http://dx.doi. org/10.3205/000103

11 American College of Chest Physicians/Society of Critical Care Medicine Consensus Conference: definitions for sepsis and organ failure and guidelines for the use of innovative therapies in sepsis. Crit Care Med 1992;20:864-74.

12 Singer M, Deutschman CS, Seymour CW, et al. The third international consensus definitions for sepsis and septic shock (sepsis-3). JAMA 2016;315:801-10.
13 Zachariasse JM, Kuiper JW, Moll HA, et al. PO-0304 safety of the Manchester Triage System in paediatric patients: does it recognise the critically ill? Arch Dis Child 2014;99 2):A343.

14 Chamberlain DJ, Willis E, Clark R, et al. Identification of the severe sepsis patient at triage: a prospective analysis of the Australasian Triage Scale. Emerg Med J 2015;32:690-7.

15 Geier F, Popp S, Greve Y, et al. Severity illness scoring systems for early identification and prediction of in-hospital mortality in patients with suspected sepsis presenting to the emergency department. Wien Klin Wochenschr 2013;125:508-15.

16 Gilboy N, Tanabe P, Travers D, et al. Emergency Severity Index (ESI): a triage tool for emergency department care, version 4. Implement Handb 2012;12-14.

17 Keep JW, Messmer AS, Sladden R, et al. National early warning score at Emergency Department triage May allow earlier identification of patients with severe sepsis and septic shock: a retrospective observational study. Emerg Med J 2016;33:37-41.

18 Norman DC. Fever in the Elderly. Clin Infect Dis 2000;31:148-51.

19 Dellinger RP, Levy MM, Rhodes A, et al. Surviving sepsis campaign: international guidelines for management of severe sepsis and septic shock, 2012. Intensive Care Med 2013;39:165-228.

20 Jefferies $S$, Weatherall $M$, Young $P$, et al. A systematic review of the accuracy of peripheral thermometry in estimating core temperatures among febrile critically ill patients. Crit Care Resusc 2011;13:194-9.

\section{IMAGE CHALLENGE}

\section{Severe chest pain in an elderly woman}

\section{CLINICAL INTRODUCTION}

A 73-year-old woman presented to the ED with non-radiating right sided chest pain, since 1 week. The pain was progressively worsening and was not associated with vomiting and there was no preceding history of trauma. She denied prior episode of chest pain. History was significant for hypertension, hyperlipidaemia and iron deficiency anaemia. There was no history of coughing or smoking. Physical examination and ECG were unremarkable. A frontal chest radiograph was obtained (figure 1).

\section{QUESTION}

Which organ is the most probable cause of chest pain, based on the radiographic findings?
A. Lung and pleura
B. Ribs
C. Oesophagus
D. Trachea

For the answer see page 248

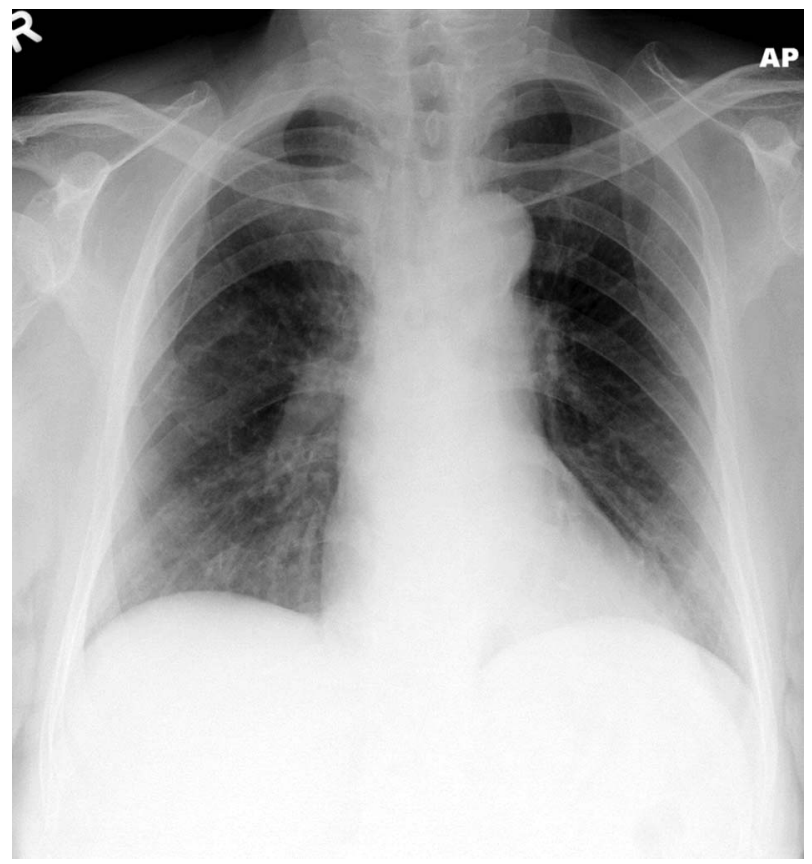

Figure 1 Frontal chest radiograph. 